\title{
Anticancer activity of natural compound (zerumbone) extracted from Zingiber zerumbet in human HeLa cervical cancer cells
}

\begin{abstract}
A natural compound, zerumbone was extracted, isolated and purified from the rhizomes of edible plant Zingiber zerumbet using methanol extraction and Column Chromatography (CC) method. The isolated and purified zerumbone crystals were subjected to High Performance Liquid Chromatography (HPLC), Liquid Chromatography Mass Spectrometry (LCMS) and 13C NMR and 1H NMR analysis to confirm the purity, molecular weight and molecular structure. The study investigated the purified zerumbone crystals for its anti-cancer properties on human cervical cancer cell line (HeLa). Cisplatin, was used as a positive control in this study. The cytotoxicity of zerumbone and cisplatin were investigated using the MTT assay and caspases-3 was estimated with colorimetric assay in zerumbone treated HeLa cells. Morphological analysis showed that there were changes observed on HeLa cancer cells after treatment with zerumbone and cisplatin. The MTT assay results demonstrated that the IC50 value $( \pm \mathrm{SEM})$ of zerumbone was determined to be $11.3 \mu \mathrm{M}(2.5 \mu \mathrm{g} \mathrm{mL}-1)$ whilst the IC50 value of cisplatin was at $7.5 \mu \mathrm{M}(1.6 \mu \mathrm{g} \mathrm{mL}-1)$. Prominent growth retardation was identified to the HeLa cancer cells, after treatment with both compounds, while caspase- 3 was observed to be significantly increased in zerumbone treated cells as compared to untreated control cells. This study showed promising avenues towards zerumbone to be developed as a new chemo-natural drug for treatment of cervical cancer.
\end{abstract}

Keyword: Zerumbone; Cervical cancer; Caspase-3; HPLC; LCMS; NMR 